\title{
Geometrical Structures of Photographic and Stereoscopic Spaces
}

\author{
Toshio Watanabe \\ Keio University, Japan
}

\begin{abstract}
Two experiments were conducted to investigate the geometrical structures of photographic and stereoscopic spaces. In Experiment 1, it was investigated how accurately photographic space reproduces real physical space, and the geometrical structure of photographic space was compared with that of visual space. As a result, the mapping function of distance between photographic and physical spaces $\left(\delta=\mathrm{ad}^{\mathrm{b}}\right)$ shows that a and b range from 0.96-1.1 and 0.69-0.78. The mapping function of angle between photographic and physical spaces $\left(\Phi=g \phi^{\mathrm{h}}\right)$ shows that $\mathrm{g}$ and $\mathrm{h}$ range from 2.37-5.29 and 0.74-0.97. Further, photographic space has larger anisotropic property than visual space and photographic space may be hyperbolic. In Experiment 2, the geometrical structure of stereoscopic space was compared with that of visual space. It was found that stereoscopic space was almost the same as visual space.
\end{abstract}

Keywords: depth perception, photograph, stereoscopic vision

Se realizaron dos experimentos para investigar las estructuras geométricas de los espacios fotográficos y estereoscópicos. En el Experimento 1 se investigó la precisión con que el espacio fotográfico reproduce el espacio físico real, y se comparó la estructura geométrica del espacio fotográfico con la del espacio visual. Como resultado la función de correspondencia para la distancia entre los espacios fotográficos y los físicos $\left(\delta=a^{\text {b }}\right)$ muestra que a y b varían entre $0.96-1.1$ y $0.69-0.78$. La función de correspondencia angular del ángulo entre los espacios fotográficos y los físicos $\left(\Phi=g \phi^{\mathrm{h}}\right)$ muestra que $\mathrm{g}$ y h varían entre 2.37-5.29 y 0.74-0.97. Además, el espacio fotográfico tiene una propiedad anisotrópica mayor que el espacio visual, y el espacio fotográfico podría ser hiperbólica. En el Experimento 2, se comparó la estructura geométrica del espacio estereoscópico con la del espacio visual. Se encontró que el espacio estereoscópico era casi igual que el espacio visual.

Palabras clave: percepción de la profundidad, fotografía, visión estereoscópica

Correspondence concerning this article should be addressed to Professor Toshio Watanabe, E-mail: watanabe@ sfc.keio.ac.jp 
The present study investigates the geometrical structures of depth indirectly perceived from photographic and stereoscopic spaces. First, we investigated how accurately photographic space reproduces the real physical space in comparison with directly observing objects in a photograph. Second, if photographic space does not reproduce the physical space accurately, we investigated how it differs from the real physical space. Specifically, we investigated the mapping function between photographic and physical spaces. Further, we investigated how accurately stereoscopic space reproduces visual space.

In the present study, the space directly perceived by observing physical space is defined as visual space, the space indirectly perceived by observing a photograph as photographic space, and the space indirectly perceived by observing stereoscopic images as stereoscopic space. As geometrical properties, an anisotropy and non-Euclidean property are discussed.

It is known that the perceived distance from a camera position to an object in a photograph is affected by the distance between an observer and the photograph. Smith (1958a) conducted an experiment in which subjects observed a photograph monocularly in two observation conditions where the distances from the observer to the photograph were $0.61 \mathrm{~m}$ and $2.9 \mathrm{~m}$. He found that the perceived distance was significantly longer in the long observation distance $(2.9 \mathrm{~m})$ than in the short observation distance $(0.69 \mathrm{~m})$. In another study (Smith, 1958b), he photographed the object (1.6-1.9 $\mathrm{m}$ in height) in the scene at different distances from the camera to the objects $(25.6$, $102.4,204.8$, and $409.6 \mathrm{~m}$ ) and asked the subjects to judge the distance from the camera position to the objects and the height of the objects in two observation distance conditions $(0.38 \mathrm{~m}$ and $1.27 \mathrm{~m})$. The result showed that the observation distance did not affect the perceived height of the object, but it did affect the perceived distance to the objects. The perceived distance was longer in the longobservation distance than in the short-observation distance. Further, Smith and Gruber (1958) studied how depth perception differed between conditions in which subjects observed the corridor of $109.7 \mathrm{~m}$ in length monocularly and in the condition in which they observed its photograph, and found that the distance perceived in the photograph was shorter than in the direct observation when subjects observed the photograph from a distance of less than $2 \mathrm{~m}$, but it was perceived longer when subjects observed the photograph from a distance of more than $2 \mathrm{~m}$. Kraft, Pattersonm, and Mitchell (1986) studied the distance from the camera position to the object and the lateral distance between objects in a photograph taken with a camera of various focal distances $(17,24,28$, and $48 \mathrm{~mm})$ and found that the perceived distance from the camera position to the object was affected by the focal distance of the camera, but the lateral distance was not. Later, Hecht, van Doorn, and Koenderink (1999) studied the distortion of perceived distance and angle in a three-dimensional scene. They photographed the corner of buildings $(16.5,67,72,108$, 113 , and $161.5^{\circ}$ ) and asked subjects to judge their angles. They found that the angle was perceived as larger both in the long-observation distance $(10 \mathrm{~m})$ and in the shortobservation distance $(1.5 \mathrm{~m})$, but the distance was perceived as longer in the short-observation distance and shorter in the long-observation distance. These studies showed that perceived distance from the camera position to the object in the photograph was longer in the long-observation distance and the perceived angle is larger both in long and short observation distances. The common features of these studies are that the subject's task was to judge the distance from the camera position to the object (egocentric distance) in the photograph, and the effect of the observation distance from the observer to the photograph was studied. The question is how the distance between two objects in the photograph is affected by the observation distance and how the visual angle subtended to the photograph is related to the perceived distance to the objects in the photograph. The present study discusses how distance between two objects in the photograph and the angle of the object from the median line are perceived.

In order to study depth perception of a photograph, it is important to study directly perceived distances and angles in observing the objects as well as the physical distances and physical angles. In the present study, the data obtained in Watanabe (2004) were used as the data of the perceived distances and angles in the direct observation (visual space). In Watanabe's study, objects $(21 \mathrm{~cm}$ in diameter and $20 \mathrm{~cm}$ in height) were placed at three locations $\mathrm{A}, \mathrm{B}$, and $\mathrm{X}$ in an open space, $21 \mathrm{~m}$ in width and $40 \mathrm{~m}$ in depth as shown in Figure 1 . The subjects were 16 undergraduate students $(8$ males and 8 females) and they observed the objects from the origin $\mathrm{O}$ binocularly. The objects $\mathrm{A}$ and $\mathrm{B}$ were fixed at $\mathrm{A}(0 \mathrm{~m}, 4 \mathrm{~m})$ and $\mathrm{B}(0 \mathrm{~m}, 14 \mathrm{~m})$ and the object $\mathrm{X}$ was placed at various locations. The distance AX was $4,10,16$, 22 , and $28 \mathrm{~m}$ and the angle BAX was $0, \pm 4, \pm 8, \pm 12, \pm 16$, and $\pm 20^{\circ}$. In all, 54 locations were used, except when object $\mathrm{X}$ coincided with object $\mathrm{B}$. The subject's task was to judge the distance $\mathrm{AX}$ as the distance $\mathrm{AB}=1$ and to judge the angle BAX at 27 locations selected randomly from 54 locations. The remaining 27 locations were presented to a

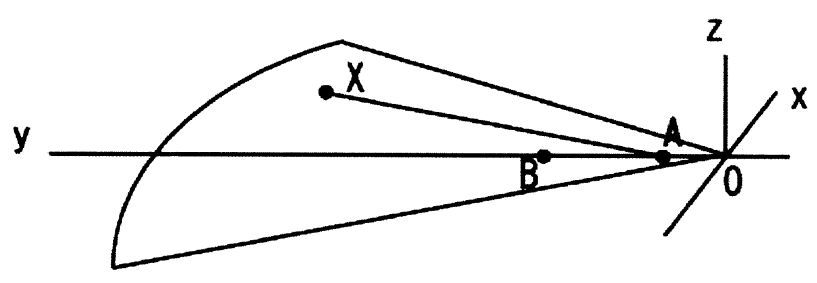

Figure 1. Illustration of experimental situation. 
different subject. Figure 2 shows the relationship between perceived distance $\mathrm{AX}$ (the perceived distance is represented as $\delta_{\mathrm{AX}}$ ) and the corresponding physical distance $\mathrm{AX}$ (the physical distance is represented as $d_{A X}$ ) when $A B=1$ in the direct observation condition (visual space). Six symbols $(\mathrm{X}, 0,+, \triangle, \square$, and $\bigcirc$ ) in the figure show the 6 angle conditions $\left(0,4,8,12,16\right.$, and $\left.20^{\circ}\right)$. Within two-factor analysis of variance (the 5-level Angle factor $\times$ the 5-level Distance factor) showed that the angle factor $(4,8,12,16$, and $\left.20^{\circ}\right)$, the distance factor $(4,10,16,22$, and $28 \mathrm{~m})$ and their interaction were all statistically significant, $F(4,28)$ $=12.29, F(4,28)=382.20, F(16,112)=3.30, p<.05$ in all cases. This result suggests that the perceived distance is affected by the angle BAX and that visual space has the property of anisotropy. In the smaller angle BAX, the distance AX is perceived as shorter. Figure 3 shows the relationship between the perceived angle BAX and the corresponding physical angle BAX. Five symbols $(0,+, \triangle$, $\square$, and $\bigcirc$ ) in the figure show the distance condition (4, $10,16,22$, and $28 \mathrm{~m}$ ). Within two-factor analysis of variance shows that the distance factor, the angle factor and their interaction are all significant, $F(4,28)=33.71$, $F(4,28)=435.71, F(16,112)=2.11, p<.05$ in all cases This result suggests that the perceived angles are affected by the distance AX, that is, geodesics AX is curved. Because the angles are perceived as larger as the distance is longer, the geodesics AX is convex on the outside. Curved geodesics supports the non-Euclidean visual space hypothesis (Blank, 1961; Indow, 1979; Luneburg, 1947). Specifically, convex geodesics suggests the hyperbolic geometry of visual space.

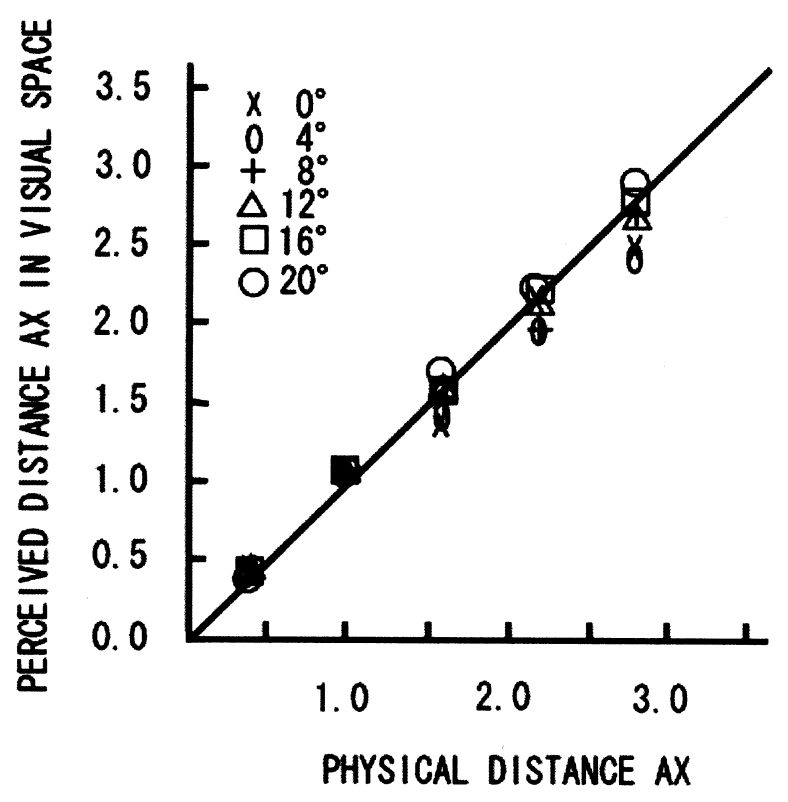

Figure 2. Relationship between perceived distances AX in visual space and their physical distances when $\mathrm{AB}=1$.
In the photographic condition of the previous study (Watanabe, 2004), the camera (Nikon DC100) was placed at the origin $\mathrm{O}$ and the three objects $\mathrm{A}, \mathrm{B}$, and $\mathrm{X}$ were photographed at 54 locations. The photograph images were projected by a digital projector (Plus U2-870) on the screen. The image size on the screen was $68 \mathrm{~cm}$ in height, $83 \mathrm{~cm}$ in width. The subject observed the 54 images on the screen $3.5 \mathrm{~m}$ in front of the screen one by one in random order. As a result, the distance $\mathrm{AX}$ in the photograph was perceived as shorter and the angle BAX was perceived as larger than in visual space. Further, a larger amount of anisotropy was observed than in visual space. In the previous study, the visual angle subtended to the scene in photographic space was different from that in visual space. As one possibility, the difference in visual angle subtended to two spaces may produce different perception between photographic and visual spaces. Therefore, in the photographic condition (Experiment 1) of the present study, the same photographs were used as in Watanabe (2004), but the size of the image projected on the screen was different from the previous study.

In stereoscopic space, various studies have been conducted (e.g., Foley, 1966, 1968, 1970; Howard \& Rogers, 1995; Julesz, 1971; Oyama, 1974). For example, Foley (1966) found that the perceptual egocentric distance lay outside the Vieth-Muller circle (VMC). This implies that stereoscopic space did not reproduce physical space accurately. These studies investigated the property of stereoscopic space with distance judgments from the subjects to the objects (egocentric distance). However, in order to investigate the geometrical structure, we should investigate not only distances

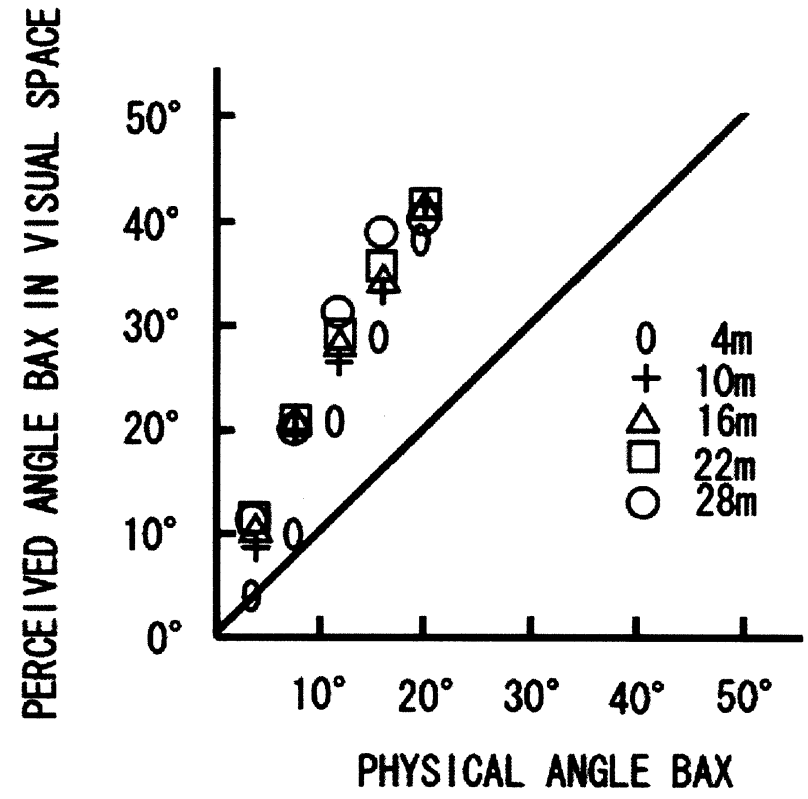

Figure 3. Relationship between perceived angle BAX in visual space and their physical angle BAX. 
but also angles. In the stereoscopic condition (Experiment 2) of the present study, inter-point distance between objects and angle were judged to investigate the geometrical structure of stereoscopic space. In Figure 1, the camera was placed at left and right eye positions of the origin $\mathrm{O}, \mathrm{L}(-3,0$, and 92 $\mathrm{cm})$, and $\mathrm{R}(3,0$, and $92 \mathrm{~cm})$. The inter-pupil distance was defined as $6 \mathrm{~cm}$.

\section{Experiment 1}

\section{Method}

\section{Participants}

Eight undergraduate students ( 3 males and 5 females) participated in Experiment 1. Their visual acuities were normal or corrected-to-normal. They were all naïve to the objectives of the experiment.

\section{Procedure}

The same photographic images were used as in Watanabe (2004). The difference in the procedure between two studies is the size of the photographic image projected on the screen and the observation distance. The subjects observed photographic images (100 cm in height and $156 \mathrm{~cm}$ in width) on the screen $1.9 \mathrm{~m}$ in front of the screen. The visual angle subtended to the photographic image was the same as that in visual space $\left(44.7^{\circ}\right)$. The subject's task was to judge the distance $\mathrm{AX}$ as the distance $\mathrm{AB}=1$ and the angle $\mathrm{BAX}$ binocularly. The order of the 54 stimuli was randomized and the subjects judged the distance AX and the angle BAX in 54 locations one time. The illuminance of the screen ranged from $10 \mathrm{~lx}$ to $20 \mathrm{~lx}$ and the room light was turned off during the experiment.

\section{Results and Discussion}

First, 50 distances and 50 angles, except angle BAX = 0 , were averaged over 8 subjects and symmetrized laterally because photographic space was isotropic in the lateral direction (Watanabe, 2004). After symmetrization, 25 distances and 25 angles were used for later analyses. Figure 4 shows the relationship of perceived distance AX between two conditions (the visual angle same as in visual space and the visual angle different from in visual space). Analysis of variance shows that there is no difference, $F(1,14)<1$. Figure 5 shows the relationship of perceived angle BAX between two conditions (the visual angle same as in visual space and the visual angle different from in visual space). Analysis of variance shows that there is no difference but there is a tendency, $F(1,14)=1.34, p<.1$. Figure 6 shows the relationship between the perceived distance $A X$ when the visual angle is the same as in visual space and the corresponding physical distance AX. The symbols used in the figure have the same meaning in Figure 2. As shown in the figure, the perceived distance $\mathrm{AX}$ becomes shorter than the corresponding physical distance AX when the corresponding physical distance becomes longer, even in the condition of the same visual angle as in visual space. Furthermore, the perceived distances AX are affected by the angle BAX. When the angle BAX changes, the perceived distance AX changes. Table 1 shows the values of parameters of $\delta=\mathrm{ad}^{\mathrm{b}}$ and root mean squares (RMS) by applying the least square method to the perceived distance $(\delta)$ and the corresponding physical distance (d) for each angle condition $\left(4,8,12,16\right.$, and $\left.20^{\circ}\right)$. The anisotropic property in visual space was found in other studies (Foley, 1966; Hagino \& Yoshioka, 1976; Higashiyama, 1992), but the important thing in the present finding is that the amount of anisotropy is larger in photographic space than in visual space. Figure 6 and Table 1 suggest that the amount of anisotropy in photographic space is larger than in visual space. Figure 7 shows the relationship between the perceived angle in Experiment 1 and the physical angle. The symbols used in the figure have the same meaning in Figure 3. The perceived angle is about twice as large as the physical angle. Furthermore, the perceived angle changes when the distance AX changes. Table 2 shows the values of parameters of $\Phi$ $=\mathrm{g} \phi^{\mathrm{h}}$ and RMS by applying least square method for the perceived angle $(\Phi)$ and the physical angle $(\phi)$ for each distance condition $(4,10,16,22$, and $28 \mathrm{~m})$. The result shows that the geodesics in photographic space is curved.

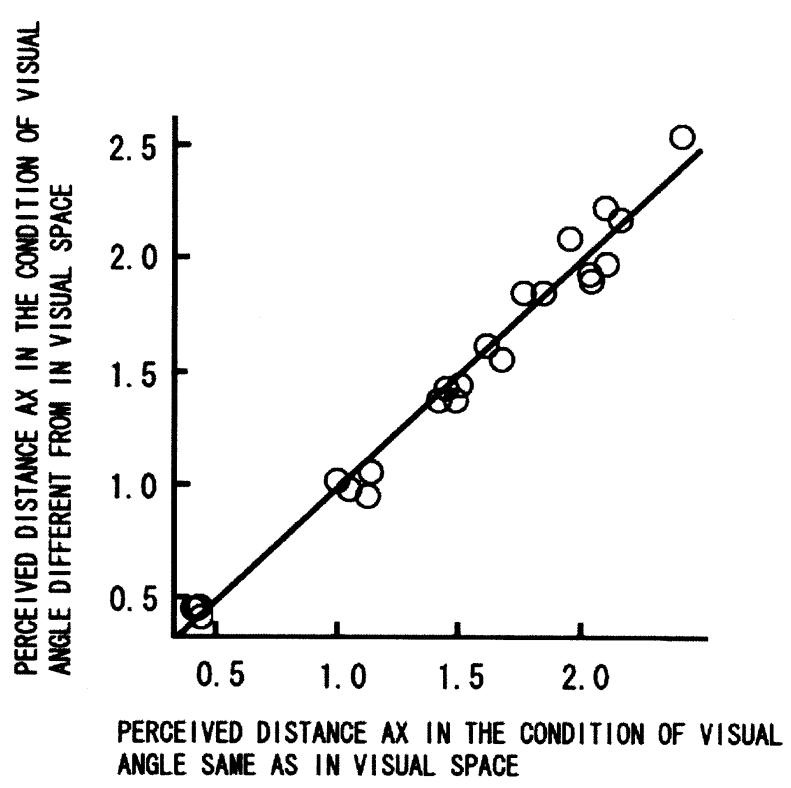

Figure 4. Relationship of perceived distance AX between two conditions (the visual angle same as in visual space and the visual angle different from in visual space). 

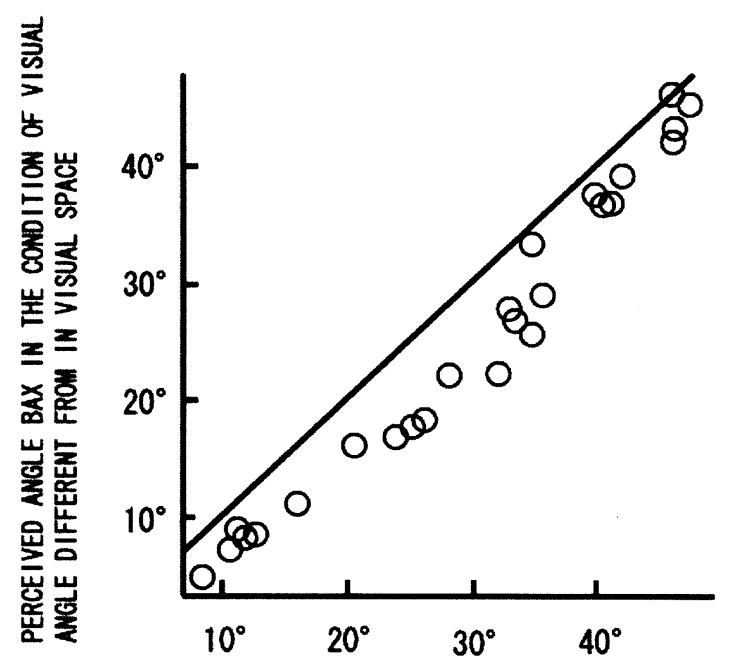

PERCEIVED ANGLE BAX IN THE CONDITION OF VISUAL ANGLE SAME AS IN VISUAL SPACE

Figure 5. Relationship of perceived angle BAX between two conditions (the visual angle same as in visual space and the visual angle different from in visual space).

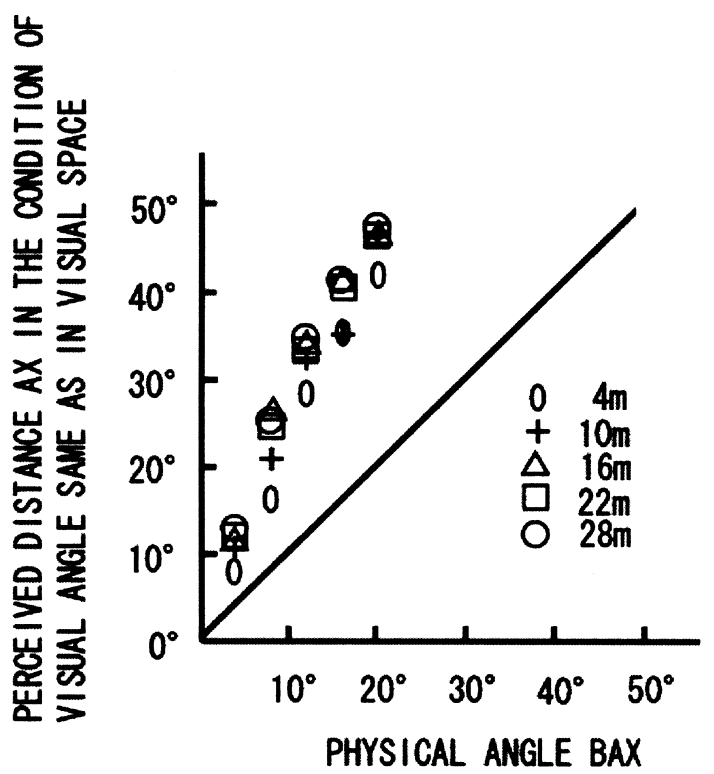

Figure 7. Relationship between the perceived angle BAX in the condition of the visual angle same as in visual space and the corresponding physical angle BAX.
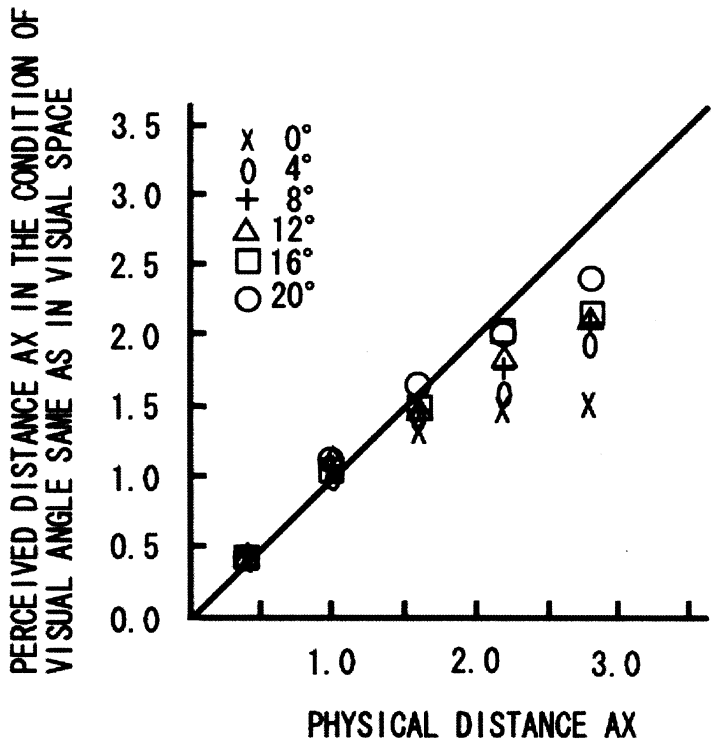

Figure 6. Relationship between the perceived distance $\mathrm{AX}$ in the condition of the visual angle same as in visual space and the corresponding physical distance AX.

Figure 8 (a) shows the physical configuration of 25 locations. This is perceived as shown in Figure 8 (b) in the same visual angle condition as in visual space. Figure 8 (b) shows that the physical lines are perceived to be curved. Furthermore, the points on the circumference of the circle whose center is point $\mathrm{A}$ are perceived as the points on the circumference of the elliptic circle whose long axis is defined along the lateral direction. This suggests that photographic space is anisotropic space in which depth direction is compressed. Furthermore, Figure 8 (c) shows the perceived configuration that is supposed to be perceived as Figure 8 (a). In order to be perceived as in Figure 8 (c), the physical configuration should be the configuration shown in Figure 8 (d). Five points along each direction shows the visual geodesics. As the geodesics in the figure is convex towards the lateral direction, it is similar to the geodesics in hyperbolic geometry. This result suggests the possibility that photographic space is hyperbolic.

Table 1

Values of Parameters of $\delta=a d^{\mathrm{b}}$ and RMS (Root Mean Squares) by Applying the Least Square Method to the Perceived Distance $(\delta)$ and the Corresponding Physical Distance $(d)$ for each Angle Condition (4, 8, 12, 16, and 20 $)$

\begin{tabular}{cccc}
\hline Angle & a & b & RMS \\
\hline $4^{\circ}$ & 0.96 & 0.69 & 0.058 \\
$8^{\circ}$ & 0.98 & 0.76 & 0.057 \\
$12^{\circ}$ & 1.03 & 0.71 & 0.069 \\
$16^{\circ}$ & 1.04 & 0.76 & 0.093 \\
$20^{\circ}$ & 1.10 & 0.78 & 0.062 \\
\hline
\end{tabular}


Table 2

Values of Parameters of $\Phi=g \phi^{h}$ and RMS by Applying Least Square Method for the Perceived Angle ( $\left.\Phi\right)$ and the Physical Angle ( $\phi)$ for each Distance Condition (4, 10, 16, 22, and $28 \mathrm{~m}$ ).

\begin{tabular}{cccc}
\hline Distance & $\mathrm{g}$ & $\mathrm{h}$ & RMS \\
\hline $4 \mathrm{~m}$ & 2.37 & 0.97 & 1.356 \\
$10 \mathrm{~m}$ & 3.56 & 0.85 & 1.801 \\
$16 \mathrm{~m}$ & 5.18 & 0.74 & 1.802 \\
$22 \mathrm{~m}$ & 4.69 & 0.77 & 1.156 \\
$28 \mathrm{~m}$ & 5.29 & 0.74 & 1.282 \\
\hline
\end{tabular}

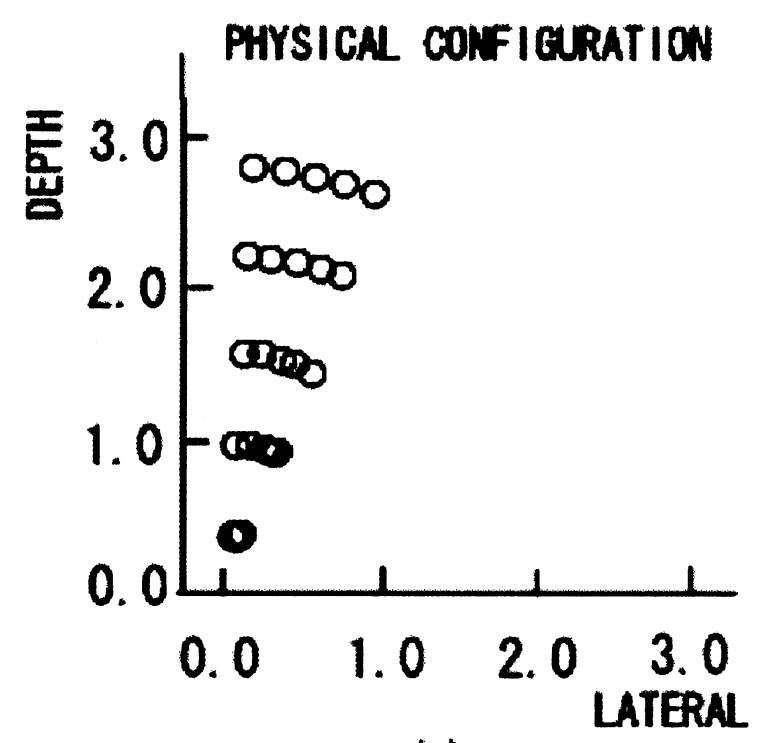

(a)

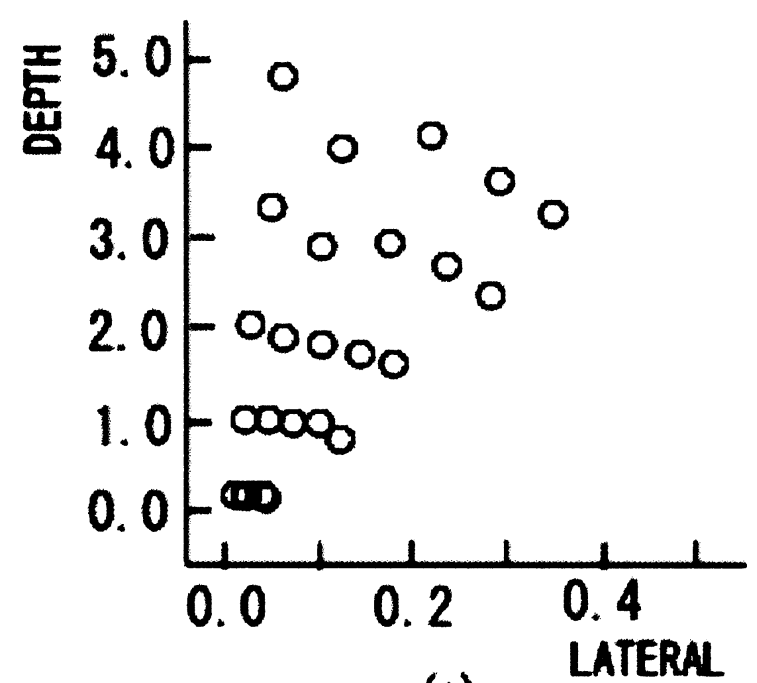

(c)
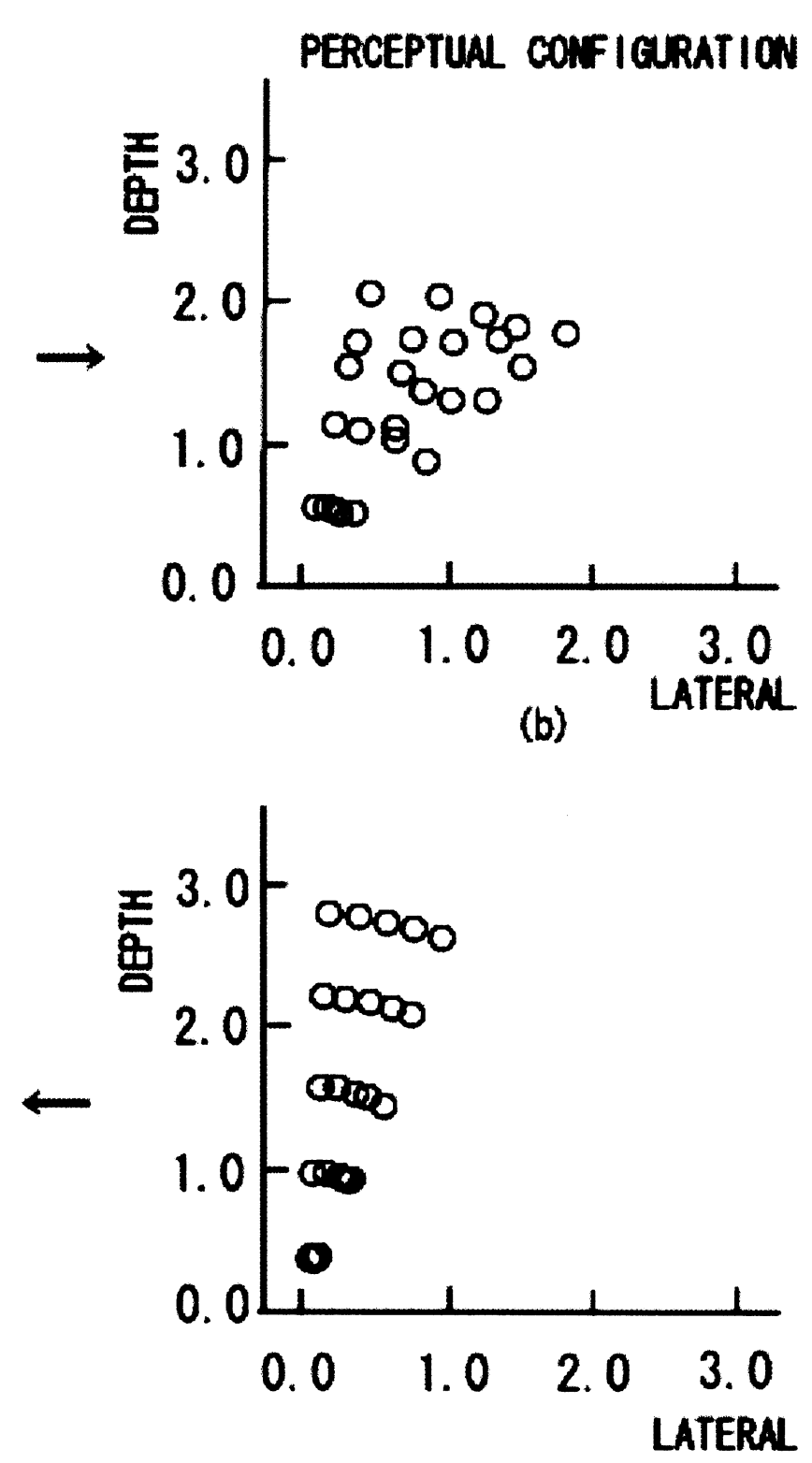

(d) 


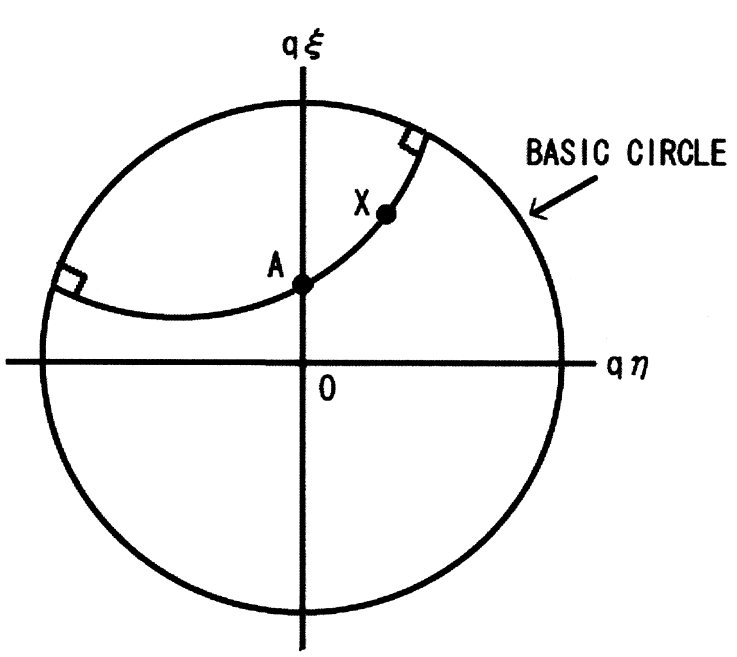

Figure 9. Hyperbolic geodesics in Euclidean map.

In the Euclidean map (Poincare's model) representing hyperbolic space, hyperbolic geodesics is represented by the Euclidean circle orthogonal to the basic circle. Therefore, hyperbolic geodesics is the circle convex to the lateral direction. The geodesics in Figure 8 (d) may correspond to the geodesics (the circle passing through points $\mathrm{A}$ and $\mathrm{X}$ ) in Figure 9. Further, the hyperbolic distance between two points $\mathrm{A}$ and $\mathrm{X}\left(\delta_{\mathrm{AX}}\right)$ is represented with the Euclidean distance $\mathrm{AX}$ in the Euclidean map $\left(\rho_{\mathrm{AX}}\right)$ in Equation 1.

$$
\begin{aligned}
& \mathrm{q} \rho_{\mathrm{AX}}=\sqrt{1-\left(\mathrm{q} \rho_{0 \mathrm{~A}}\right)^{2}} \sqrt{1-\left(\mathrm{q} \rho_{0 \mathrm{X}}\right)^{2}} \sinh \left(\mathrm{q} \delta_{\mathrm{AX}}\right) \\
& \mathrm{q}=\sqrt{-\mathrm{K} / 2}
\end{aligned}
$$

In the equation, $\mathrm{K}$ shows the value of the curvature. In the case of hyperbolic circle of radius $\mathrm{R}$, whose center is located at $\mathrm{A}, \mathrm{q} \rho_{0 \mathrm{~A}}, \sinh \left(\mathrm{q} \delta_{\mathrm{AX}}\right)$ are constant.

Furthermore,

$$
\begin{aligned}
& \mathrm{q} \rho_{\mathrm{AX}}=\sqrt{\left(\mathrm{q} \xi_{\mathrm{A}}-\mathrm{q} \xi_{\mathrm{X}}\right)^{2}+\left(\mathrm{q} \eta_{\mathrm{A}}-\mathrm{q} \eta_{\mathrm{X}}\right)^{2}} \\
& \mathrm{q} \rho_{0 \mathrm{X}}=\sqrt{\left(\mathrm{q} \xi_{\mathrm{X}}\right)^{2}+\left(\mathrm{q} \eta_{\mathrm{X}}\right)^{2}}
\end{aligned}
$$

From Equations 1 and 2, we obtain:

$$
\begin{aligned}
& \left(\mathrm{q} \xi_{\mathrm{X}}-\mathrm{q} \xi_{\mathrm{A}} /(1+\mathrm{F})\right)^{2}+\left(\mathrm{q} \eta_{\mathrm{X}}-\mathrm{q} \eta_{\mathrm{A}} /(1+\mathrm{F})\right)^{2}= \\
& \mathrm{F}\left(1-\left(\mathrm{q} \rho_{0 \mathrm{~A}}\right)^{2}+\mathrm{F}\right) /(1+\mathrm{F})^{2}
\end{aligned}
$$

where $\mathrm{F}=\left(1-\left(\mathrm{q} \rho_{0 \mathrm{~A}}\right)^{2}\right) \sinh ^{2}\left(\mathrm{q} \delta_{\mathrm{AX}}\right)$.

Equation 3 suggests that hyperbolic circle is represented by Euclidean circle in the Euclidean map. Next, in the Euclidean map representing elliptic space, elliptic geodesics (the circle passing through points $\mathrm{A}$ and $\mathrm{X}$ ) is represented by the Euclidean circle whose two intersections of the basic circle are symmetric about the origin $\mathrm{O}$ as shown in Figure 10. Therefore, elliptic geodesics is represented by the circle

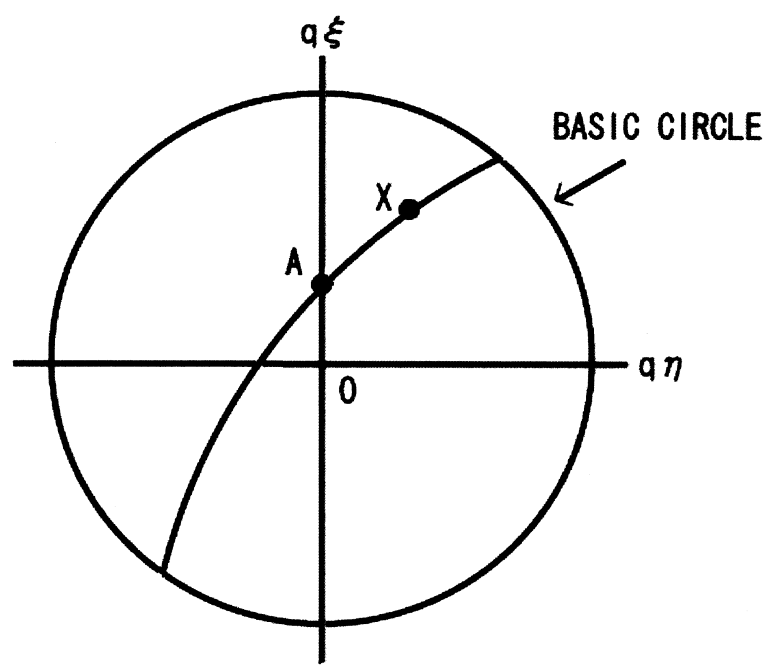

Figure 10. Elliptic geodesics in Euclidean map.

convex towards the depth direction ( $\xi_{\text {axis }}$ ). Further, the elliptic distance $\mathrm{AX}\left(\delta_{\mathrm{AX}}\right)$ is represented with the Euclidean distance $\mathrm{AX}\left(\rho_{\mathrm{AX}}\right)$ in the following equation:

$$
\begin{aligned}
& \mathrm{q} \rho_{\mathrm{AX}}=\sqrt{1+\left(\mathrm{q} \rho_{0 \mathrm{~A}}\right)^{2}} \sqrt{1+\left(\mathrm{q} \rho_{0 \mathrm{X}}\right)^{2}} \sin \left(\mathrm{q} \delta_{\mathrm{AX}}\right) \\
& \mathrm{q}=\sqrt{\mathrm{K} / 2}
\end{aligned}
$$

In the equation, $\mathrm{K}$ shows the value of the curvature. In the case of elliptic circle of radius $\mathrm{R}$, whose center is located at $\mathrm{A}, \mathrm{q} \rho_{0 \mathrm{~A}}, \sin \left(\mathrm{q} \delta_{\mathrm{AX}}\right)$ are constant. Therefore,

$$
\begin{aligned}
& \mathrm{q} \rho_{\mathrm{AX}}=\sqrt{\left(\mathrm{q} \xi_{\mathrm{A}}-\mathrm{q} \xi_{\mathrm{X}}\right)^{2}+\left(\mathrm{q} \eta_{\mathrm{A}}-\mathrm{q} \eta_{\mathrm{X}}\right)^{2}} \\
& \mathrm{q} \rho_{0 \mathrm{X}}=\sqrt{\left(\mathrm{q} \xi_{\mathrm{X}}\right)^{2}+\left(\mathrm{q} \eta_{\mathrm{X}}\right)^{2}}
\end{aligned}
$$

From Equations 4 and 5, we obtain:

$$
\begin{aligned}
& \left(\mathrm{q} \xi_{\mathrm{X}}-\mathrm{q} \xi_{\mathrm{A}} /(1-\mathrm{F})\right)^{2}+\left(\mathrm{q} \eta_{\mathrm{X}}-\mathrm{q} \eta_{\mathrm{A}} /(1-\mathrm{F})\right)^{2}= \\
& \mathrm{F}\left(1+\left(\mathrm{q} \rho_{0 \mathrm{~A}}\right)^{2}-\mathrm{F}\right) /(1-\mathrm{F})^{2},
\end{aligned}
$$

where $\mathrm{F}=\left(1+\left(\mathrm{q} \rho_{0 \mathrm{~A}}\right)^{2}\right) \sin ^{2}\left(\mathrm{q} \delta_{\mathrm{AX}}\right)$.

This suggests that elliptic circle is represented by the Euclidean circle in the Euclidean map. Figure 11 shows the relationship between the perceived distance in the condition of the same visual angle as in visual space (Experiment 1) and the perceived distance in visual space (Watanabe, 2004). From the figure, even in the same visual angle condition, the distance is not accurately reproduced, $F(1,14)=5.87, p<$ .05. Figure 12 shows the relationship between the perceived angle in the condition of the same visual angle condition as in visual space (Experiment 1) and the perceived angle in visual space (Watanabe, 2004). The analysis of variance shows there is no significant difference, $F(1,14)=2.72, p>.05$. That is, the same visual angle condition reproduces the angle accurately. As a result, the perceived angle changes when the 


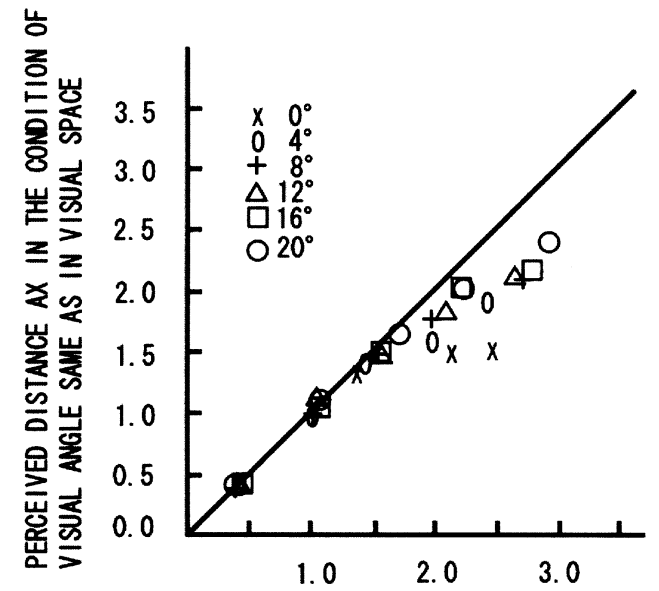

PERCEIVED DISTANCE AX IN VISUAL SPACE

Figure 11. Relationship between the perceived distance in the condition of the same visual angle as in visual space and the perceived distance in visual space (Watanabe, 2004).

visual angle subtended to the photograph changes. This suggests that photographic space has non-Euclidean property. In the non-Euclidean geometries such as hyperbolic or elliptic geometry, similar figures do not exist. Therefore, photographic space is affected by the photographic size (visual angle).

\section{Experiment 2}

\section{Participants}

Nine undergraduate students (5 males and 4 females) participated in the experiment. Their visual acuities were normal or corrected to normal.

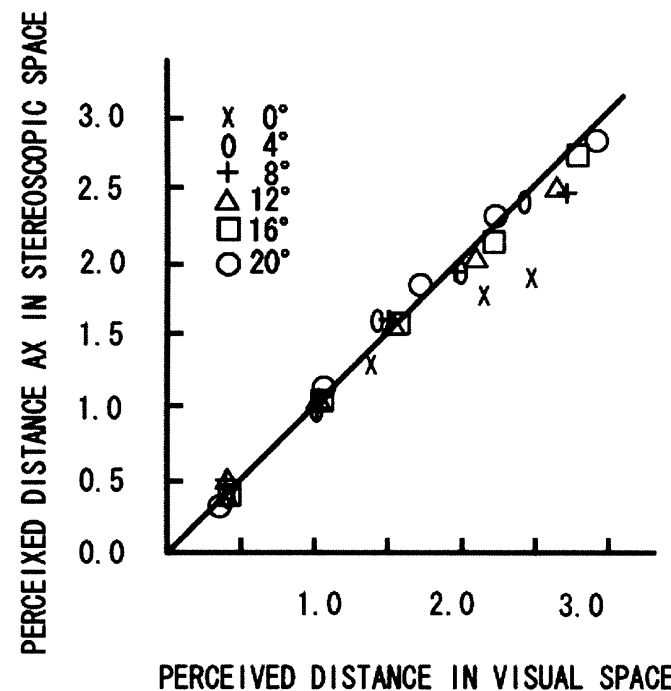

Figure 13. Relationship between visual distances in the stereoscopic space and visual distances in visual space.

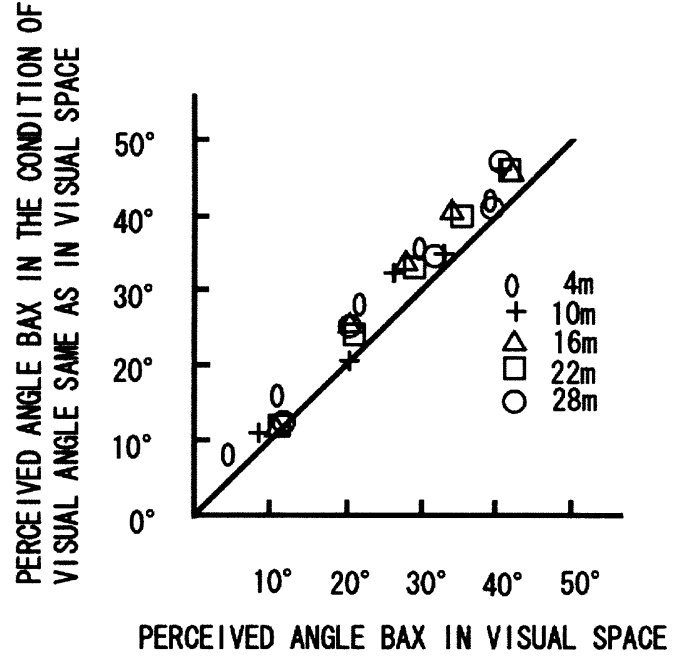

Figure 12. Relationship between the perceived angle BAX in the condition of the same visual angle condition as in visual space (Experiment 1) and the perceived angle BAX in visual space (Watanabe 2004).

\section{Procedure}

In two locations, $\mathrm{L}(-3,0$, and $92 \mathrm{~cm})$ and $\mathrm{R}(3,0$, and $92 \mathrm{~cm}$ ), shown in Figure 1, a digital camera was placed and the objects A, B, and X at 54 locations were photographed. The distance of finders of two cameras is $6 \mathrm{~cm}$ and this corresponds to the average inter-pupil distance. Photographic images were projected by two projectors on the screen. A polarized filter was placed on the lens of each projector. The distance between the projector and the screen was $4.4 \mathrm{~m}$ and two projectors were placed at the same position, one on top of the other. The projector projecting the right image was placed on the bottom and the projector projecting the left

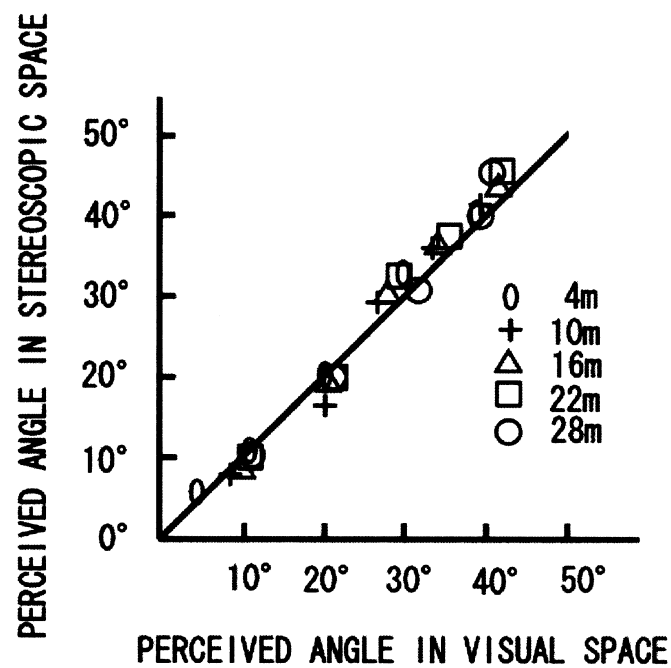

Figure 14. Relationship between perceived angle in stereoscopic space and perceived angle BAX in visual space 
image on the top. The lateral distance between two lenses was $6 \mathrm{~cm}$. The size of the image on the screen was $100 \mathrm{~cm}$ in height and $156 \mathrm{~cm}$ in width. Right eye image was projected $6 \mathrm{~cm}$ to the right of the left eye image. The subject observed the images with polarized glasses binocularly 1.9 $\mathrm{m}$ in front of the screen. The visual angle subtended to the images was the same visual angle in visual space. The illuminance of the screen ranged from $10 \mathrm{~lx}$ to $20 \mathrm{~lx}$ and the room light was turned off. The subject's task was to judge the distance $\mathrm{AX}$ as the distance $\mathrm{AB}=1$ and the angle $\mathrm{BAX}$ once at 54 locations (4 locations at the center, 25 locations at the left, and 25 locations at the right side). The distance $\mathrm{AX}$ was $4,10,16,22$, and $28 \mathrm{~m}$ and the angle BAX was 0 , $\pm 4, \pm 8, \pm 12, \pm 16$, and $\pm 20^{\circ}$. Figure 13 shows the relationship between the perceived distance in stereoscopic space and in visual space. There was no significant difference, $F(1,15)$ $<1$. Figure 14 shows the relationship between the perceived angle in stereoscopic space and in visual space. There was no significant difference, $F(1,5)<1$. As a result, stereoscopic space preserves the distance and the angle perception in visual space. Stereoscopic space reproducing the same depth perception as in visual space makes a large contribution to space perception.

\section{General Discussion}

In the present article, we discussed depth perception in photographic and stereoscopic spaces. The main purpose of the study was to determine how accurately photographic space reproduces physical space in comparison with visual space, and how accurately stereoscopic space reproduces visual space. As a result, it was found that photographic space could not reproduce the real physical space and, further, it could not reproduce the perceived depth in visual space even from the same visual angle condition as visual space. The distance in photographic space was perceived as shorter than in physical and visual spaces when physical distance was large. In the mapping function of distance between photographic and physical spaces $\left(\delta=\mathrm{ad}^{\mathrm{b}}\right)$, the value of a ranges from $0.96-1.1$, and the value of $b$ ranges from 0.69-0.78. The values of $a$ and $b$ become larger when the angle BAX becomes larger. And, the angles were perceived larger than in physical and visual spaces. In the mapping function of angle between photographic and physical spaces $\left(\Phi=\mathrm{g} \phi^{\mathrm{h}}\right)$, the value of $\mathrm{g}$ ranges from 2.37-5.29 and the value of $h$ ranges from $0.74-0.97$. The value of $\mathrm{g}$ becomes larger when the distance $\mathrm{AX}$ becomes longer, but the value of $h$ becomes smaller when the distance AX becomes longer. Further, a larger amount of anisotropic property than in visual space and in the curved geodesics property were found in photographic space. These results suggest the possibility that photographic space is hyperbolic. When the size of the photograph is different, photographic space tends to be perceived differently. This may stem from the non-Euclidean property of photographic space because in non-Euclidean space, such as hyperbolic space and elliptic space, there is no similar figure. If the size of the figure is enlarged or shrunken in hyperbolic or elliptic space, the shape of the figure changes. In other studies (Smith, 1956a, 1965b; Smith \& Gruber, 1958), it was found that the observation distance affected the perceived distance. When the observation distance changes, the size of the image may change. Under such a condition, the above finding will occur.

Hagen (Hagen, 1974; Hagen \& Glick, 1977; Hagen, Jones, \& Reed, 1978) found the distortion of the distance and the size of the object in pictorial space, and Finch (1977) found that hyperbolic projection gave better depth effect in drawing. The present study may support Finch's finding. If photographic space is hyperbolic, a similar property will be found in pictorial space. Therefore, the hyperbolic projection will emphasize depth in drawing. In Gibson's study (Gibson, 1951), the texture gradient plays an important role in depth perception. As one possibility, space drawn with texture gradient may also be hyperbolic. The study of photographic space will make various contributions to the study of pictorial space and space drawn with texture gradient.

In other studies on stereoscopic space, it did not reproduce physical space accurately.

However, in the present study, stereoscopic space accurately reproduced depth in visual space in the same visual angle condition. The difference in the result stems from various factors. For example, in the present study, stereoscopic space was compared with visual space instead of physical space. It is important to investigate whether stereoscopic vision differs from visual space perception. Even if stereoscopic space does not reproduce physical space accurately, if it reproduces visual space accurately, it plays an important role. The present result showed that stereoscopic space can be used as a substitute for physical space, at least in the depth range of $28 \mathrm{~m}$. This makes a significant contribution to the study of space perception. By editing the stereoscopic image, we can study space perception, which cannot be in real physical space. For example, we can edit the size of objects in an image and this gives us the opportunity to study the effect of object size in depth perception.

\section{References}

Blank, A.A. (1961). Curvature of binocular visual space. An experiment. Journal of the Optical Society of America, 51, 335-339.

Finch, D. (1977). Hyperbolic geometry as an alternative to perspective for constructing drawings of visual space. Perception, 6, 178-182.

Foley, J.M. (1966). Locus of perceived equi-distance as a function of viewing distance. Journal of the al Society of America, 56, 822-827. 
Foley, J.M. (1968). Depth, size and distance in stereoscopic vision. Perception \& Psychophysics, 3, 265-274.

Foley, J.M. (1970). Loci of perceived, equi-, half- and doubledistance in stereoscopic vision. Vision Research, 10, 1201-1209.

Gibson, J.J. (1951). A theory of pictorial perception. Audio-Visual Communication Review, 1, 1-23.

Hagen, M.A. (1974). Picture perception: Toward a theoretical model. Psychological Bulletin, 81, 471-497.

Hagen, M.A., \& Glick, R. (1977). Pictorial perspective: Perception of size, linear, and texture perspective in children and adults. Perception, 6, 675-684.

Hagen, M.A., Jones, R.K., \& Reed, E.S. (1978). On a neglected variable in theories of pictorial perception: Truncation of the visual field. Perception \& Psychophysics, 23, 326-330.

Hagino, G. \& Yoshioka, I. (1976). A new method for determining the personal constants in the Luneburg theory of binocular visual space. Perception \& Psychophysics, 19, 499-509.

Hecht, H., van Doorn, A., \& Koenderink, J.J. (1999). Compression of visual space in natural scenes and in their photographic counterparts. Perception \& Psychophysics, 61, 1269-1286.

Higashiyama, A. (1992). Anisotropic perception of visual angle: Implications for the horizontal-vertical illusion, overconstancy of size, and the moon illusion. Perception \& Psychophysics, $51,218-230$.

Howard, I.P. \& Rogers, B.J. (1995). Binocular vision and stereopsis. New York: Oxford University Press.
Indow, T. (1979). Alleys in visual space. Journal of Mathematical Psychology, 19, 221-258.

Julesz, B. (1971). Foundations of cyclopean perception. Chicago: University of Chicago Press.

Kraft, R.N., Patterson, J.F., \& Mitchell, N.B. (1986). Distance perception in photographic displays of natural settings. Perceptual and Motor Skills, 62, 179-186.

Luneburg, R.K. (1947). Mathematical analysis of binocular vision. Princeton, NJ: Princeton University Press.

Oyama, T. (1974). Perceived size and perceived distance in stereoscopic vision and an Analysis of their causal relations. Perception \& Psychophysics, 16, 175-181.

Smith, O.W. (1958a). Comparison of apparent depth in a photograph viewed from two distances. Perceptual and Motor Skills, 8, 79-81.

Smith, O.W. (1958b). Judgments of size and distance in photographs. American Journal of Psychology, 71, 529538.

Smith, O.W., \& Gruber, H. (1958). Perception of depth in photographs. Perceptual and Motor Skills, 8, 307-313.

Watanabe, T. (2004). Anisotropy in depth perception of photograph. The Journal of Psychology, 75, 24-32.

Received December 15, 2005

Review received April 19, 2006 Accepted May 1, 2006 